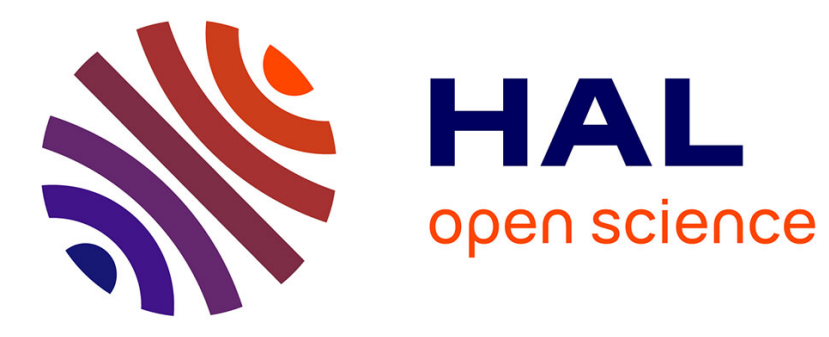

\title{
Vehicular radio connectivity in urban environment
}

Kahina Ait-Ali, Alexandre Gondran, Alexandre Caminada, Laurent Moalic

\section{To cite this version:}

Kahina Ait-Ali, Alexandre Gondran, Alexandre Caminada, Laurent Moalic. Vehicular radio connectivity in urban environment. ISWCS 2010, 7th International Symposium on Wireless Communication Systems, Sep 2010, York, United Kingdom. pp 305-309, 10.1109/ISWCS.2010.5624296 . hal-00938423

\section{HAL Id: hal-00938423 \\ https://hal-enac.archives-ouvertes.fr/hal-00938423}

Submitted on 16 May 2014

HAL is a multi-disciplinary open access archive for the deposit and dissemination of scientific research documents, whether they are published or not. The documents may come from teaching and research institutions in France or abroad, or from public or private research centers.
L'archive ouverte pluridisciplinaire HAL, est destinée au dépôt et à la diffusion de documents scientifiques de niveau recherche, publiés ou non, émanant des établissements d'enseignement et de recherche français ou étrangers, des laboratoires publics ou privés. 


\title{
Vehicular Radio Connectivity in Urban Environment
}

\author{
Kahina Ait Ali ${ }^{\# 1}$, Alexandre Gondran ${ }^{* 2}$, Alexandre Caminada ${ }^{\# 3}$, Laurent Moalic ${ }^{\# 4}$ \\ \# UTBM, SET, Belfort, France \\ ${ }^{1}$ kahina.ait-ali@utbm.fr \\ ${ }^{3}$ alexandre.caminada@utbm.fr \\ ${ }^{4}$ laurent.moalic@utbm.fr \\ *Ecole Nationale de l'Aviation Civile, Toulouse, France \\ ${ }^{2}$ alexandre.gondran@enac.fr
}

\begin{abstract}
In this paper we present a study of mobile connectivity for vehicular network in urban environment. The considered area is a real map divided into equally sized cells, each characterized by a set of information including the type of structure in the cell, cell altitude and cell attraction for vehicles. These characteristics are taken into account in two models: vehicular mobility model and electromagnetic wave propagation model. We study the impact of nodes density and obstacles present in the environment on network connectivity based on radio links. Graphs representing radio connections between vehicles are periodically generated. We examine four metrics that are nodes degree, connections duration, dominated nodes rate and cluster formation.
\end{abstract}

\section{INTRODUCTION}

In recent years, several researches have been conducted on inter-vehicle communication systems. According to that one infrastructure is used or not, two communication types may be distinguished: Vehicle-to-Infrastructure (V2I) communications where vehicles are connected to stations located on roadsides for information acquisition, information transmission and internet access; and Vehicle-to-Vehicle (V2V) communications, in which vehicles exchange information without relying on any given infrastructure, they collaborate to form at each time a dynamic distributed system called VANET (Vehicular Ad hoc NETwork).

In ad hoc networks, the possibility of establishing communications and the quality of exchanged messages depend on the radio connectivity between nodes. One factor that affects considerably this connectivity is nodes motion, particularly when nodes move at high speeds as it is the case for vehicular networks. Several models have been proposed to simulate nodes mobility in MANET [1]. These models ignore the key characteristics of vehicular traffic, mainly the constraints on nodes movement and the interactions between neighboring nodes; they are thus not suitable for VANET. Aiming to reflect as closely as possible the real behavior of vehicular traffic, new models have been proposed [2]. Depending on the level of detail considered, these models may be classified into two categories: macroscopic models that take into account parameters such as road topology, roads attributes, traffic signs and traffic characteristics (density, flow etc.) and microscopic models that focus on the individual behavior of each vehicle and try to model features such as speed, acceleration, braking and interactions between vehicles.
The second factor which plays an important role on network connectivity is the environment in which radio signals propagate. Indeed, signal deteriorations depend on the type and the density of physical obstacles present in the environment. The effect of these obstacles must be taken into account in one radio wave propagation model. However the majority of studies done on ad hoc networks neglects this parameter and assumes a flat surface where transmission range is modeled by an ideal circle around each node, then radio propagation is constant which is absolutely wrong.

The evaluation of the impact of parameters such as nodes density, nodes velocity and obstacles on network connectivity is a good starting point for analysis, improvement or development of routing strategies. However, there are few studies in the literature on radio connectivity in VANET. The authors of [3] considered an unobstructed highway and studied the impact of transmission range on routes lifetime. In [4] vehicles are assumed to travel at their maximum velocities in an unobstructed highway. The authors examined the effect of density, velocity and distance between communicating vehicles on connections lifetime. The considered environment in [5] is an urban environment where vehicles move in a road topology extracted from a real city map. The authors analyzed the average nodes degree for various traffic flow condition, transmission range and time interval. They also presented an algorithm for computing the transitive connectivity. To demonstrate the impact of employed mobility model on vehicular network topology, Fiore and Härri [6] studied the network connectivity under various mobility models based on different approaches and simulated vehicular motion with different levels of realism. The authors considered a simple grid road topology and examined link duration, nodal degree and network clustering.

In this paper we present a study of vehicular network connectivity in a real urban environment. Unlike the above mentioned works that assume an unobstructed environment and model the communication range by a simple circle around the transmitter, we define and use a propagation model to capture obstacles effect on radio signals. The considered city map is divided into cells of equal size each characterized by information representing its ability to attract vehicles, its altitude and type of structure located in the cell. This information is taken into account in both mobility and propagation models. We consider different traffic densities and examine nodes degree, connections lifetime, dominated 
nodes rate and cluster formation. To show the importance of taking into account the impact of obstacles on radio signal propagation, we also evaluate these metrics assuming an unobstructed flat environment.

The remainder of this paper is organized as follows: Section 2 presents the propagation model and its adaptation to our case study. Simulation environment and results are presented in Section 3. Finally, Section 4 concludes the paper.

\section{Propagation Model}

The propagation model employed in our simulations is based on the statistical path loss model defined in [7] for suburban environments. This model uses empirically measured data collected across the United States in a large number of existing macrocells that cover a wide range of terrain types. It contains three terrain categories: category A that corresponds to a hilly terrain with moderate-to-heavy trees densities, this category has the maximum path loss values; the middle category, $\mathrm{B}$, characterized as either mostly flat terrain with moderate-to-heavy trees densities or hilly terrain with light tree densities; the last category, $\mathrm{C}$, is mostly flat terrain with light tree densities, lowest path loss values are obtained with this category. The path loss formula is:

$$
\begin{gathered}
P L=A+10 \gamma \log _{10}\left(d / d_{0}\right)+s \quad d \geq d_{0} \\
A=20 \log _{10}\left(4 \pi d_{0} / \lambda\right) \\
\gamma=\left(a-b h_{b}+c / h_{b}\right)+x \sigma_{\gamma} \\
s=y\left(\mu_{\sigma}+z \sigma_{\sigma}\right)
\end{gathered}
$$

$d$ is the distance between the base antenna and the mobile antenna, $A$ is a fixed quantity corresponding to the free-space path loss where $d_{0}=100 \mathrm{~m}$ and $\lambda$ is the wavelength in meters. The path loss exponent $\gamma$ is a Gaussian random variable over the population of macrocells within each terrain category. In (3) $h_{b}$ is base station antenna height in meters, $x$ is a zeromean Gaussian variable of unit standard deviation, $\mathrm{N}[0,1]$ and $a, b, c$ and $\sigma_{\gamma}$ are data-derived constants for each terrain category. $s$ is the shadow fading component, it varies randomly from one location to another within any given macrocell; $y$ and $z$ are both zero-mean Gaussian variables of unit standard deviation, $\mathrm{N}[0,1]$ and $\mu_{\sigma}$ and $\sigma_{\sigma}$ are dataderived constants for each terrain category.

We did improvement to this model to adapt it to our environment and to our numerical data. The environment considered in our study is a real city map (urban environment). The area is divided into equally sized cells each characterized by its altitude and the percentage of each type of structure presents in the cell (e.g. $20 \%$ of building with height $<10,10 \%$ forest...). Each cell is at first classified as building or forest by comparing the percentage of forest to the sum of percentages of building located in the cell. Categorization according to the path loss model is determined as follows: all cells with a percentage of building less than $33 \%$ belong to category $\mathrm{C}$, those with a percentage between $33 \%$ and $66 \%$ belong to category $\mathrm{B}$ and the rest of cells fall into category A. The path loss is computed at the center of each cell, the obtained value is assumed the same throughout the cell. Only cells located at a distance less than $d_{\max }$ from the transmitter are considered. Beyond this distance the received signal strength is assumed null. To compute the path loss between two cells $G_{t}$ (transmitting antenna location) and $G_{r}$ (receiving antenna location) the total distance $d_{t}$ crossed by a signal over each terrain category situated between $G_{t}$ and $G_{r}$ is determined. The news path loss formulas are:

$$
\begin{aligned}
& P L= \begin{cases}A+10 \gamma \log _{10}\left(d / d_{0}\right)+s & d \geq d_{0} \\
20 \log _{10}(4 \pi d / \lambda)+s & d<d_{0}\end{cases} \\
& \gamma=\frac{1}{d} \sum_{t=1}^{T} \gamma_{t} d_{t} \\
& \mu_{\sigma}=\frac{1}{d} \sum_{t=1}^{T} \mu_{\sigma t} d_{t} \\
& \sigma_{\sigma}=\frac{1}{d} \sum_{t=1}^{T} \sigma_{\sigma t} d_{t}
\end{aligned}
$$

$\gamma, \mu_{\sigma}$ and $\sigma_{\sigma}$ are the averages computed over all terrain categories located between $G_{t}$ and $G_{r}$ and $T \in[1,3]$ is the number of these categories. The height difference $h_{b}$ between transmitting and receiving antenna is calculated taking into account cells altitude (altitude of $G_{t}$ and $G_{r}$ ).

\section{SIMULATION AND RESULTS}

We used the application "Territoire Mobile" for simulation. This application represents the city of Belfort in the north-eastern of France. Several data have been used to reproduce the real environment including GIS shapefiles representing the city map, survey data and socio-economical information collected by professionals for regional planning needs. The area is divided into square cells, each characterized by the types of structure located in the cell (roads, buildings, forest...), cell altitude and cell attraction power for vehicles.

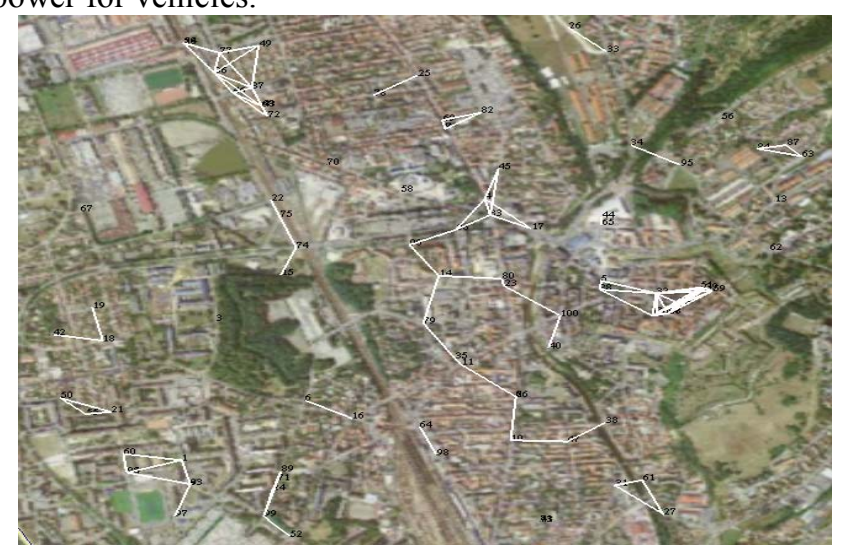

Fig. 1. Initial connection graph for 100 vehicles distributed in an area of $2500 \mathrm{~m} * 2500 \mathrm{~m}$.

The mobility model we used is V-MBMM (VehicularMask Based Mobility Model) [8] which is a combination of MBMM [9][10] and IDM (Intelligent Driver Model). This model simulates vehicles displacement in urban area taking into consideration the characteristics of the environment. A full description of V-MBMM is in [8]. The propagation 
model described in Section 2 has been integrated to the application by considering only the cells which are on roads. In other words, the path loss is only computed for road cells but it takes into account all obstacles between transmitters and received even outside the roads.

An undirected graph $G(V, E, t)$ is generated every $T$ seconds. The set $V$ of vertices represents vehicles and an edge $e_{i j}$ links two vertices $v_{i}$ and $v_{j}$ belong to $E$ if vehicle $i$ is in the transmission range of vehicle $j$ and vice versa. Figure 1 shows an example of a graph obtained with 100 vehicles.

\section{A. Metrics}

In all simulations we vary the nodes density and from the generated graphs we estimate four metrics that are:

- Nodes degree. It is an important indicator of the possibility or not to establish communication sessions between nodes in ad hoc networks. The average degree of node $n_{i}$ over all graphs is:

$$
d\left(n_{i}\right)=\frac{1}{N} \sum_{k=1}^{N}\left|N g b_{i k}\right|
$$

where $N$ is the total number of graphs generated during simulation and $N g b_{i k}$ is the set of all nodes connected to a node $n_{i}$ in a graph $k$.

- Connections lifetime. This metric provides information on network stability. Low values indicate that network topology is frequently changing. For more precision, instead of computing only a single value that represents the average connection duration, we define intervals of increasing duration and compute for each one the rate of connections whose duration value belongs to the interval. An age value is maintained for each connection. The age is initialized to the value $T$ when the connection appears. For each new generated graph, we check if the connection still exists. If so the value $T$ is added to age, otherwise the number of connections of the interval that contains age value is incremented by 1 .

- Dominated nodes rate. This metric gives a general idea on the rate of redundancy and thus the probability of collisions that may occur in the network mainly because of broadcast transmissions of data or control packets. This can be very common in VANET with high nodes density and vehicles queuing at road intersections. We consider that a node $n_{i}$ is dominated by its neighbor $n_{j}$ if all the neighbors of node $n_{i}$ are also neighbors of node $n_{j}$. Therefore, messages dissemination by node $n_{i}$ may be unnecessary.

- Clustering. In vehicular networks, entities tend to move in groups formed mainly because of vehicles stop at intersections and traffic congestion on roads. This phenomenon favors the use of hierarchical routing strategies instead of a flat routing in order to limit messages dissemination in the network. Thus, the latter factor we analyze is the formation of clusters in the network. We define a cluster as a set of nodes connected to a master node (cluster head) and whose the number of hops from that master is less than a predefined value Hpmax. To determine a cluster, we consider at each step all the nodes that are not part of any cluster and select as a cluster head the node with the maximum number of neighbors. This is achieved by the following algorithm:

\section{Clustering Algorithm}

\section{Variables:}

$V$ : set of simulated nodes and CS: set of identified clusters.

$c$ : current cluster and $c h$ : node selected as a cluster head.

$A S$ : the set in which the algorithm selects the members of $c$.

$N_{S}\left(n_{i}\right)$ : the set of neighbors of node $n_{i}$ in the set $S$.

Hpmax: maximum number of hops from the cluster head to any

other member of the cluster.

$\operatorname{NbHp}\left(n_{i}, n_{j}\right)$ : number of hops from node $n_{i}$ to node $n_{j}$.

Algorithm:

$C S:=\Phi, A S:=V$;

while $\exists n_{i} \in A S /\left|N_{A S}\left(n_{i}\right)\right|>0$

begin

$c:=\Phi$

ch $:=n_{i} \in A S /\left|N_{A S}\left(n_{i}\right)\right|=\max \left\{\left|N_{A S}\left(n_{j}\right)\right| n_{j} \in A S, j=1 . .|A S|\right\}$

$c:=c \cup N_{A S}(c h) \cup\{c h\}$;

$A S:=A S / c$

while $\exists n_{k} \in c /\left(\left|N_{A S}\left(n_{k}\right)\right|>0\right.$ and $\operatorname{NbHp}\left(c h, n_{k}\right)<$ Hpmax $)$

begin

$$
c:=c \cup N_{A S}\left(n_{k}\right)
$$

end

$$
A S:=A S / N_{A S}\left(n_{k}\right) \text {; }
$$

end

$$
C S:=C S \cup c
$$

When the value of Hpmax is set to infinity this algorithm returns the isolated groups of nodes in the network that we denote in the remainder of this paper by fragment.

\section{B. Results}

Simulations were performed on an area of $2500 \mathrm{~m} * 2500 \mathrm{~m}$ divided into cells of $25 \mathrm{~m} * 25 \mathrm{~m}$. We vary the number of vehicles from 50 to 400 and set the maximum speed to $15 \mathrm{~m} / \mathrm{s}$. The numerical values of all constants related to the terrain categories contained in the path loss model are the same as in [7]. We set $h_{b}$ to $1 \mathrm{~m}, d_{\max }$ to $500 \mathrm{~m}$ and the frequency to $5,9 \mathrm{Ghz}$ as specified in $802.11_{\mathrm{p}}$ norm. To show the impact of obstacles on network connectivity, we also estimate the four metrics by considering circular transmission ranges of $200 \mathrm{~m}$ radius. All simulations run for 3,600 seconds, the measurements are recorded after the first 900 seconds to exclude initiation phase. Connection graphs are updated every one second. In the remainder of this paper we use the notation $T C$ (Terrain Characteristics) to refer to the propagation model that takes into account terrain characteristics and the notation TR (Transmission Range) in reference to the propagation model that uses simple circles as transmission ranges.

Figures 2 and 3 depict vehicles percentage versus average degree values obtained respectively with TR and TC. As expected, the results show that the average degree increases when vehicular density grows. By comparing the curves obtained with TC to those of TR we can notice that ignorance of obstacles impact leads to optimistic results on nodes connections. With TC, the percentage of isolated nodes, when the number of simulated vehicles is 400 , is $3 \%$, this value increases with decreasing node density to reach $35 \%$ when the density is 50 vehicles. With TR, the percentages are 
respectively $1 \%$ and $25 \%$. From the figures it can also be observed that for small average degree values TC presents higher percentages. However, for high values (above 2, 3, 6 and 10 respectively for a density of 50,100, 200 and 400 vehicles) results obtained with TR are higher. In general and as it can also be seen in Figure 4 that shows the average degree value for all simulated nodes, the results obtained with TR are optimistic that is the connections between nodes are less important so communications are more difficult than estimated.

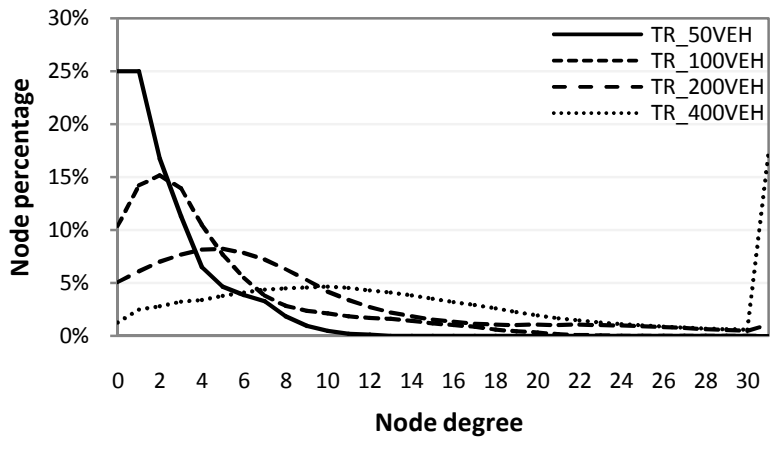

Fig. 2. Nodes average degrees distribution obtained with TR

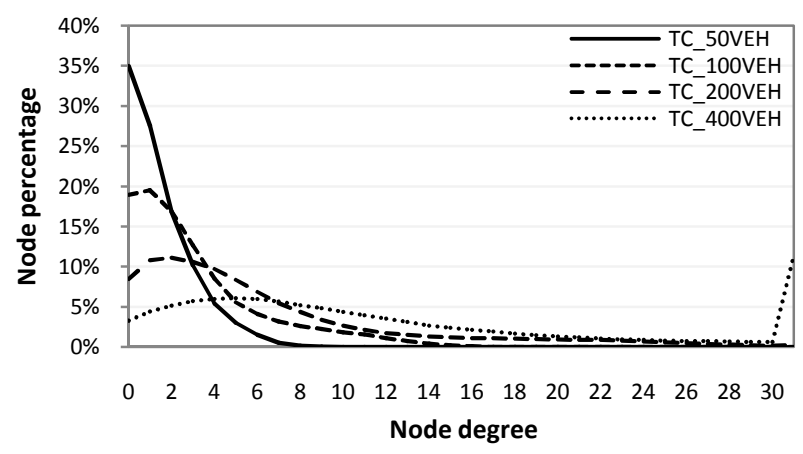

Fig. 3. Nodes average degrees distribution obtained with TC

Measurements on connections lifetime are depicted in Figures 5 and 6 . The duration intervals, in seconds, considered in simulations are $[0,4[,[4,10[,[10,20[\ldots$ Each interval is represented on the graph by its upper bound. Both figures show that the density has almost no effect on connections lifetime. The reason is that no congestion situation occurs for all considered densities and thus the average velocity is almost the same. Therefore we discuss two factors that are the effect of obstacles on connections lifetime and connections lifetime distribution regardless of nodes density.

As for the average degree, it can be observed that the results obtained with TR are higher, too optimistic, compared to those with TC. Figure 6 shows that the average connections lifetime value obtained with TR (48 seconds) is the double of that obtained with TC ( 24 seconds). With TR the connectivity is only affected by vehicle displacements, however, with TC the effect of real obstacles is also taken into account. The results on average connections lifetime indicate that the impact of obstacles is as important as that of mobility (lifetime halved). When focusing on the connections lifetime

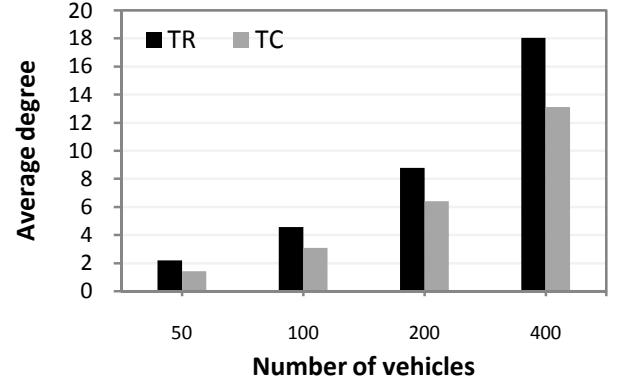

Fig. 4. Average nodes degree vs. Nodes density

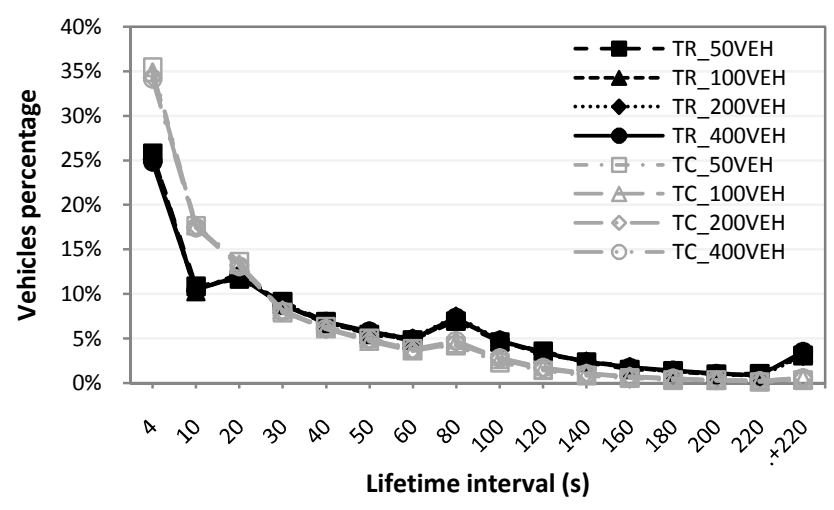

Fig. 5. Connections lifetime distribution

distribution, it can be seen from Figure 5 that more than $63 \%$ of connections with TR and $65 \%$ with TC have the duration less than the average value. The curves show that about $25 \%$ of connections with TR and $35 \%$ with TC have a lifetime value not exceeding 4 seconds. This indicates that network topology is continuously changing but much more than expected with circle range.

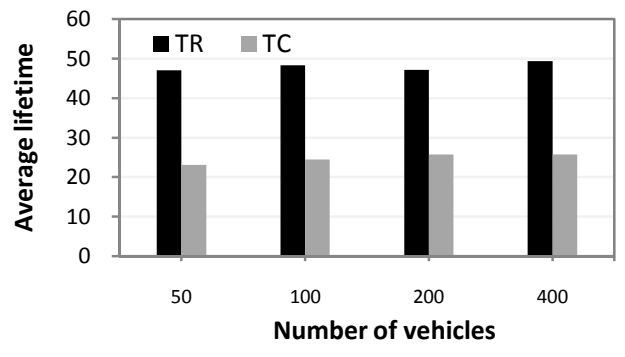

Fig. 6. Average connections lifetime vs. Nodes density

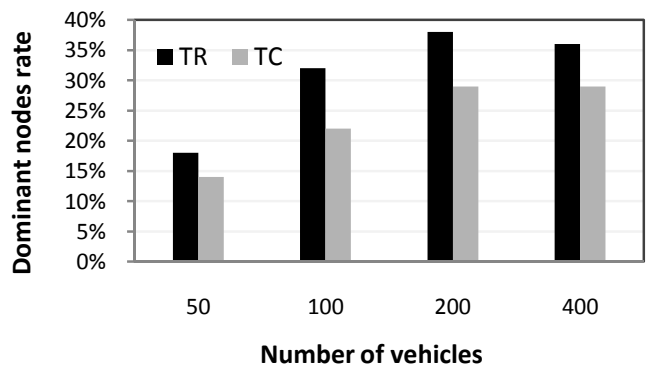

Fig. 7. Dominated nodes rate vs. Nodes density 
The dominated nodes rate obtained for all densities values, from 50 up to 400, is given in Figure 7. It can be observed that the values are quite high. With TR, at a density of 50 vehicles the number of dominated nodes is 9; this value increases when vehicular density grows to reach 144 for a density of 400 vehicles. With TC, the number of dominated nodes, for each density, is smaller compared to that obtained with TR; it is 7 and 116 for the densities of 50 and 400 vehicles respectively.

We did another series of tests to study the clustering formation in the network. We considered two values of Hpmax. In order to analyze network fragmentation, we first set Hpmax value to infinity. In the second series of tests we limited the number of hops from the cluster head to 4 . The results are shown in Figures 8 and 9. The notation FRG refers to the fragmentation and CLS to clusters with Hpmax set to 4 . We can observe that at low density of vehicles, the results obtained with TR are almost equal to those obtained with TC; the difference becomes significant when vehicular density grows. Figure 8 shows that at low density, that is 50 vehicles, the number of fragment is almost equal to the number of clusters. The reason is that given the low nodes density, the maximum number of hops from the cluster head in each fragment does not exceed 4 and therefore the clusters formed with Hpmax set to 4 are all isolated groups of nodes. Obviously, the average number of nodes per cluster is in this case equal to the average number of nodes per fragment. Increasing vehicle density magnifies the difference between

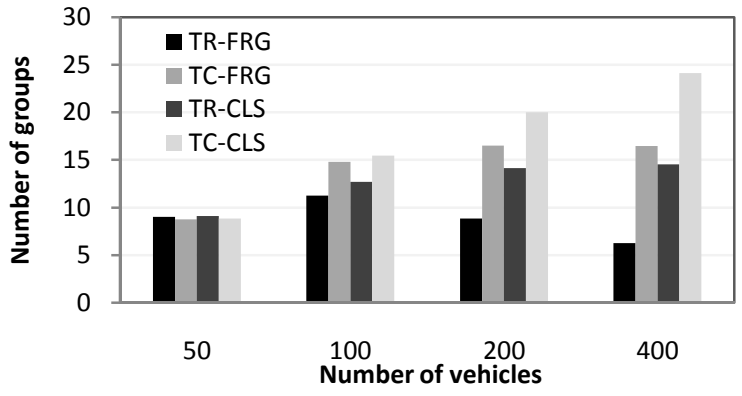

Fig. 8. Number of groups vs. Vehicles density

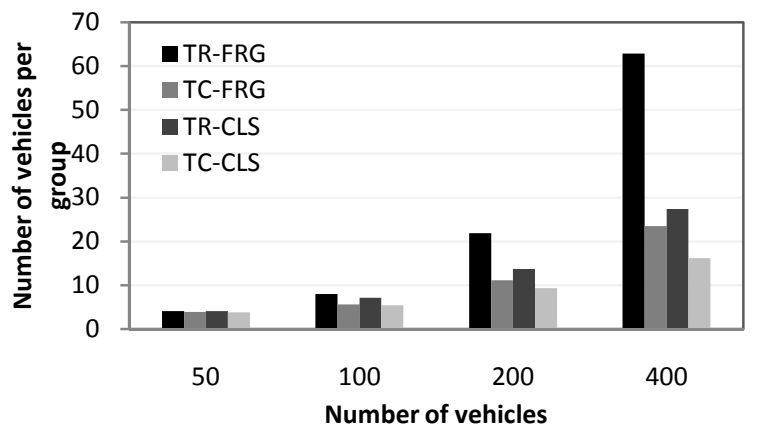

Fig. 9. Average number of vehicles per group

clusters and fragments. At high densities the network becomes more connected leading to larger fragments in terms of number of hops from the cluster head and average number of nodes per fragment. With TR, for a density of 400 vehicles, the number of fragments is 6.27 and the average number of nodes per fragment is 62.88. The number of clusters is much higher; it is 14.53 with an average number of nodes per cluster equal to 27.38. With TC the number of fragments (clusters) is 16.48 (24.13) and the number of nodes per fragment (cluster) is 23.49 (16.16). Everything concludes that VANET cannot be simulated with confidence without a right modeling of connection. Most of time the error ratio on connection features is equal to 2 .

\section{CONCLUSION}

In this paper we presented a study of mobile connectivity for vehicular network in an urban environment. We evaluated 4 metrics that are nodes degree that gives information on the possibility or not to establish communication between remote nodes, connections lifetime that indicates the frequency of network topology change, dominated nodes rate whose objective is to give an approximation on messages redundancy and the cluster formation which demonstrates that nodes in vehicular network move in groups. We have also shown the importance of taking into account the impact of obstacles on radio signals propagation by considering two propagation models. The first one reflects the characteristics of the environment by defining three terrain categories according to obstacles density; radio signals are more attenuated in the presence of obstacles. The second model assumes an unobstructed flat space and represents node transmission range by a simple circle. The study has shown that when obstacles are ignored, the results are really optimistic with an over connection of ratio 2 in comparison with reality.

\section{REFERENCES}

[1] T. Camp, J. Boleng, and V. Davies, "A survey of mobility models for ad hoc network research," WCMC: Special issue on Mobile Ad Hoc Networking: Research, Trends and Applications, vol. 2, no. 5, pp. 483-502, August 2002.

[2] J. Härri, F.Filali, and C. Bonnet, "Mobility Models for Vehicular Ad Hoc Networks: A Survey and Taxonomy," Institut Eurecom. Rep. 06$168,2007$.

[3] S.Y. Wang, "The Effects of Wireless Transmission Range on Path Lifetime in Vehicle-Formed Mobile Ad Hoc Networks on Highways,' in Proc. IEEE ICC'05, 2005, p. 3177.

[4] M.M. Artimy, W. Robertson and W.J. Phillips, "Connectivity in intervehicle ad hoc networks," in Proc. IEEE CCECE'04, 2004, pp. 293298.

[5] H. Conceição, M. Ferreira and J. Barros, "On the Urban Connectivity of Vehicular Sensor Networks," in Proc. IEEE DCOSS'08, 2008, pp 112-125

[6] M. Fiore and J. Härri, "The Networking Shape of Vehicular Mobility," in Proc. ACM MobiHoc, 2008, p. 261.

[7] Erceg, L.J. Greenstein et al "An Empirically Based Path Loss Model for Wireless Channels in Suburban Environments,". IEEE Journal on Selected Areas of Communications., vol. 17, pp. 1205-1211, July1999.

[8] K. Ait Ali, M. Lalam, L. Moalic and O. Baala, "V-MBMM: Vehicular Mask-based Mobility Model," in Proc. ICN'10, 2010, p.243.

[9] C. Joumaa, A. Caminada and S. Lamrous, "Mask Based Mobility Model A new mobility model with smooth trajectories," SpaSWiN'07, 2007

[10] C. Joumaa, A. Caminada and S. Lamrous, "Mask Based Mobility Model: A 2-D indoor 3-D outdoor mobility model," EMC'07, 2007.

[11] M. Trieber, A. Hennecke and D. Helbing, "Congested traffic states in empirical observations and microscopic simulations," Phys. Rev. E 62, Issue 2, August 2000. 